\title{
Tracking genetic discrimination
}

The promise of personalized medicine lies in the tailored treatment of individual patients, a process requiring detailed phenotypic and genetic information. Although the widespread collection of such data can help to advance the implementation of precision healthcare, the genomic sequencing data being amassed also include private information that could potentially be used as a basis for genetic discrimination. It is important for the genetics community to be aware of these risks and to contribute to policies designed to monitor and mitigate threats to the equitable treatment of individuals or populations on the basis of genetics.

A $\mathrm{s}$ is often the case when novel technologies develop quickly, the ethical, legal and societal implications of the resultant newly obtained information are not always fully considered or explored. Scientific progress can outpace humanity's ability to responsibly cope with the consequences of technological advancement. In the human genetics field, the stakes are particularly high, because of the risks posed by increased availability of, and access to, sensitive genomic data. Misuse of such data could lead to exclusionary or harmful practices manifesting in various forms of genetic discrimination (for example, genetic data being inappropriately handled by insurance companies or law enforcement). Therefore, the community must develop strategies to define, monitor and reduce instances of genetic discrimination on a global scale.

In this month's issue of Nature Genetics, the International Genetic Discrimination Observatory presents their initiative to track and analyze genetic-discrimination events reported from around the world. The goal of this initiative is to provide useful resources to the community for the assessment and prevention of genetic discrimination. With a membership panel comprising experts from 19 jurisdictions, this consortium aims to bring diverse knowledge and broad perspective to the complex topic of genetic discrimination, and the online platform will serve as an updated source for the latest information, including a real-time genetic-discrimination map showing worldwide approaches to genetic-discrimination policy, the incidence of genetic discrimination in life-insurance studies and laws against genetic discrimination in US states. Interestingly, one feature allows the public (currently those in the United States and Canada, although expansion to other locations is planned) to 'report a case' of genetic discrimination. These data will be aggregated and harmonized for analysis and used to shape policies intended to protect individuals or groups from becoming victims of genetic discrimination. Finally, the International Genetic Discrimination Observatory will serve as an educational resource for the public, raising awareness by making information and tools available for a non-specialist audience.

It is helpful to discuss and establish a definition of genetic discrimination, which is something of an umbrella term that refers to any use of genetic information to justify unequal treatment. This characterization obviously covers genetic information (such as genomic DNA sequencing) but can also encompass non-genetic information, such as DNA-methylation data (which could potentially be used to calculate an 'aging' signature). As new genomic technologies develop, we must consider not only how they can be utilized to benefit humanity but also how they could potentially be misused, and ensure that appropriate protections are put in place.

It is likewise important to think about how issues relating to data privacy and genetic discrimination might affect different groups of people, including patients, scientists, research participants and customers of direct-to-consumer genetic-testing services. Having policies that protect against discrimination based on genetics would make genome sequencing less of a risk. This decreased risk could in turn increase participation in research or improve patient care through using genetic medicine to implement targeted therapeutics.

Your DNA is a precious resource that should not be exploited or used as a basis for unequal treatment. It is a universal human right, as stated more than 20 years ago in The United Nations Educational, Scientific and Cultural Organization (UNESCO) Universal Declaration on the Human Genome and Human Rights: "no one shall be subjected to discrimination based on genetic characteristics." Being vigilant about where and how genetic discrimination occurs will help us to combat it and allow for its eventual eradication.

Published online: 6 May 2020 https://doi.org/10.1038/s41588-020-0632-3 